\title{
„Ich benutze tatsächlich viele Zeichen aus der Musik."
}

JENNY SCHRÖDL IM GESPRÄCH MIT DER SCHAUSPIELERIN LISAN LANTIN

JENNY SCHRÖDL: Lisan, herzlichen Dank, dass Du heute Zeit gefunden hast für dieses Interview im Rahmen unseres Sammelbandes „Phänomen Hörbuch. Interdisziplinäre Perspektiven und medialer Wandel“. An diesem Projekt „Hörbuch“, welches Stephanie Bung und Nicole Dehé 2012 initiiert haben, hast Du von Anfang an teilgenommen: Du warst bereits beim ersten Workshop in Konstanz (2012) und bei der zweitägigen Tagung in Berlin (2015) dabei. Bei letzterer hast Du Dich gemeinsam mit Deinem Kollegen Christian Wincierz mit einer spannenden Lesung („Medley aus Texten und Liedern von Hoffmann, Kaléko, Williams und Moers“) beteiligt - und ich frage Dich heute auch nicht als Absolventin der Theaterwissenschaft, sondern eben als Schauspielerin und als Praktikerin. Was hast Du als Schauspielerin eigentlich mit Hörbüchern zu tun?

LisAn LANTIN: Mit Hörbüchern direkt hat man als Schauspielerin erst einmal nichts zu tun. Das ist ein Berufsfeld, in das man sich unter anderem auch vorwagen kann. In der Ausbildung geht es ausschließlich um Theater und an gewissen Schulen auch um Film. Aber die Arbeit mit Text oder mit Sprache ist natürlich essentieller Bestandteil unserer Arbeit, also eigentlich unser täglich Brot. Aber ich habe zum Beispiel als Berufseinsteigerin eine Rolle gesprochen im Hörspiel „It Must be so", einem Hörbuch über Richard Wagner, bei dem Jens Neubert die Regie geführt hat. Tessa Mittelstaedt und Katharina Thalbach haben unter anderem ebenfalls mitgesprochen. Für mich war dabei total neu, dass man die Dialoge gar nicht mit seinem Partner aufnimmt, sondern jeder einzeln. Man muss sich ganz auf den Regisseur verlassen, dass das, was der einem sagt, auch stimmt, also man kann nicht vom anderen abnehmen im Dialog. Das war für mich eine ganz neue 
Erfahrung, das hatte ich mir anders vorgestellt. Und Katharina Thalbach und Tessa Mittelstaedt habe ich auch nicht kennengelernt. Leider!

JS: Das ist also ein recht einsames Sprechen, ohne Kopräsenz der anderen Schauspieler oder Schauspielerinnen sowie des Publikums. Was würdest Du generell sagen, sowohl als Schauspielerin als auch als Privatperson, was macht Dein Interesse am Hörbuch aus?

LL: Ich liebe Hörbücher, und zwar schon immer. Schon als Kind habe ich wahnsinnig gerne vorgelesen bekommen und auch Kassetten gehört. Die Form des EtwasErzählt-Kriegens ist so schön. In meiner Schauspiel-Diplomarbeit, die nicht so wissenschaftlich sein musste wie an Universitäten, habe ich auch über die Arbeit des Schauspielers als Sprecher geschrieben, über Sprache und Sprechproduktion. Über den Vorgang, wie das anatomisch funktioniert, aber auch wie man das schafft, die Bedeutungsebene, welche in der Stimme immer noch drin liegt, bewusst zu lenken, wie kann man das einsetzen, was gibt es für Techniken? Und am Ende kommt heraus, dass man es nicht weiß. Alle machen es irgendwie anders.

JS: Dein Schauspielstudium hast Du in Rostock absolviert, welche Rolle und Bedeutung nimmt eine Sprechausbildung im Schauspielstudium ein?

LL: Eine große Rolle, also wahrscheinlich ein Drittel. Ich erhebe keinen Anspruch auf komplette Richtigkeit, aber man macht ungefähr ein Drittel Szenenstudienarbeit, das heißt Rollenstudien zu bestimmten Epochen, bestimmte Stücke mit Partnern erarbeiten usw. Dann hat man ein Drittel Arbeit am Körper, also Tanzen, Akrobatik, Fechten, Steppen, Bewegungsunterricht, Koordination, Rhythmus, Pantomime, Bühnenkampf usw. Und eben schließlich ein Drittel Sprech- und Sprachausbildung, wobei ich auch die Gesangsausbildung mit dazu zähle, weil am Ende schauspielerisches Singen immer ein Sprechen auf Ton ist. Wir hatten, glaube ich, zwei Stunden Einzelunterricht Sprechen und Sprecherziehung jede Woche. Am Anfang gab es das sogenannte Gruppensprechen, wo man dann in einer Gruppe Übungen gemacht hat, bestimmte Dinge erlebt, seine Hörgewohnheiten geschärft hat usw. Das wurde später abgelöst durch das Körper-Stimm-Training. Dann haben wir zusätzlich Diktion unterrichtet bekommen. Begonnen haben wir mit Prosa, später kam dann Lyrik. Es ging um die Fragen: Wie geht man mit gebundener Sprache um, wie geht man mit bestimmten Sprachformen um, wie kann man eine gute stimmliche Übersetzung finden und eine gute spielerische Haltung dazu entwickeln? Und wir haben am Anfang Theorie des Sprechens gehabt, da ging es auch einfach um die anatomischen Voraussetzungen, wie der Körper funktioniert, welche Einstellungsmöglichkeiten wir haben, um eine Resonanz zu erzeugen und laut 
zu werden, was ist eine Atemstütze etc. Das musste man erst einmal verstehen, weil es danach oft leichter ist, es anzuwenden, wenn man verstanden hat, wie es funktioniert.

JS: Und welche Rolle spielen zum Beispiel Aufnahmeformate und Medien?

LL: Mikrofon-Sprechen haben wir überhaupt nicht gelernt. Das ist tatsächlich etwas, das fehlt, weil es auch ein Arbeitsbereich ist, der immer größer wird. Sowohl im Synchronsprechbereich, wozu auch Computerspiele oder Animationsfilme gehören, als auch im Audio-Bereich, also Hörbücher, Radio, Rundfunk-Features u.a. Das ist ein Berufsfeld, das in der Ausbildung nicht berücksichtigt wird; und das, obwohl das Mikrofon-Sprechen eigentlich noch mal etwas Anderes erfordert als Bühnen-Sprechen oder Sprechen in der Kammer, also in einem kleineren Raum.

JS: Das Mikrofon-Sprechen wird ja aber auch auf der Bühne immer wichtiger. Kaum eine theatrale Inszenierung verzichtet heute auf die vielfältigen technischen Mittel. Allerdings - und es wäre interessant zu erfahren, was Du dazu denkst - haben wir, und insbesondere Vito Pinto, in unserem SFB-Projekt „Stimmen als Paradigmen des Performativen“ (SFB 447 „Kulturen des Performativen“, FU Berlin, 2002-10) die These verfolgt, dass Mikrofon und Mikroport auf der Bühne weniger als bloße Hilfsmittel oder gar Prothesen zur besseren Verständlichkeit, Verstärkung oder Schonung der Stimme eingesetzt werden, sondern vielmehr als eigenständige ästhetische Mittel. Mit dem Einsatz von Mikrofonen werden beispielsweise Verfremdungseffekte erzeugt, die die Aufmerksamkeit und Wahrnehmung auf das Hier und Jetzt der stimmlichen Verlautbarung lenken. Etwas, das in unserer vorwiegend visuellen Kultur durchaus nichts Selbstverständliches ist. Zudem werden mit der Trennung von Körper und Stimme durch die Mikrofone Fragen der Identität, der Räumlichkeit, aber auch der Ortlosigkeit der Stimme aufgeworfen. Wie schätzt Du die Bedeutung der Mikrofone im zeitgenössischen Theater ein? Spielen solche Fragen in die Arbeit als (Bühnen-)Schauspielerin hinein?

LL: Ein Stück weit sicher - es hängt immer davon ab. Üblicherweise werden zum Beispiel Mikroports immer bei Kindervorstellungen in großen Häusern eingesetzt, zum Beispiel beim klassischen Weihnachtsmärchen; diese werden dann auch dazu benutzt, um Effekte zu machen. Wobei ich mir da nicht ganz sicher bin, ob es ausschließlich um die Effekte geht. Der Einsatz der Mikroports hat sicherlich ebenfalls damit zu tun, dass man in den Weihnachtsmärchen viel singt und dann einfach entweder Live-Musik oder Playback hat, über die man drüber kommen muss. 
JS: Also spielt das Mikroport doch nur als Hilfsmittel für beispielsweise die Steigerung der Lautstärke eine Rolle?

LL: Nein, sicher nicht ausschließlich. Im Abendspielplan hat es immer eine Bedeutung, wenn man an ein Mikrofon geht, mit einem Mikrofon spricht. Und es macht stets einen anderen Raum auf. Ich habe gerade eine sehr tolle Lesung zum Thema Pussy Riots gemacht im Rahmen von den Festtagen „Unbeschreiblich weiblich“ der TUP in Essen. Und hier haben wir fast nur Texte von Nadeschda Andrejewna Tolokonnikowa aus ihrem Buch „Anleitung zu einer Revolution“ benutzt. Und zwischendurch haben wir weitere Texte eingesetzt von zum Beispiel Olympe de Gouges, Simone de Beauvoir, Lady Bitch Ray. Diese Texte haben wir immer ins Mikrofon gesprochen, so dass es wie ein anderer Ort erscheint, also ein Fremdtext, den man eben dadurch besonders kennzeichnet. Und ich glaube, nur zur Unterstützung der Lautstärke braucht man in den Schauspielhäusern, die wir in Deutschland haben, keine Mikrofone.

JS: Worin liegen für dich die Unterschiede - aber auch Gemeinsamkeiten - zwischen einer Rede auf der Bühne (ob nun freie Rede oder szenische Lesung) und einer Lesung im Rahmen eines Hörbuchs?

LL: Eine Gemeinsamkeit ist, egal, ob man einen Text auswendig kann, ob man ihn spielt, ihn abliest, oder ob man ihn einfach nur zur Verfügung stellt, dass man immer eine Klarheit in der gedanklichen Führung braucht. Also, das Klassische: was sagt man, wie, wann, warum, zu wem, wo und wozu? Das muss man immer wissen. Und ein Unterschied zwischen Lesung und Bühnensituation ist natürlich, dass man bei einer Bühnensituation den Text auswendig kann, bei einer Lesung nicht. Daraus ergibt sich dann der Vorgang des Ablesens und der Kommunikation mit dem Publikum: Wann schau ich auf das Papier, wann schau ich ins Publikum? Wie gehe ich mit den Blättern in der Hand um? Wie verhält sich mein Körper dazu? Und bei einem Hörbuch kommt eben noch das Mikrofon dazu.

JS: Und wenn Du jetzt nochmal die Live-Situation und die Studio-Situation vergleichst, gibt es da eklatante Unterschiede oder verschwimmen die Sprech-Situationen dann schon eher?

LL: Doch, es ist schon ein großer Unterschied, ob ein Publikum da ist oder man ein Mikrofon vor sich hat. Weil das Publikum reagiert und es entsteht wirklich ein Austausch, so dass sich Sachen verändern können während des Vortrags. Man reagiert auf das, was das Publikum macht. Wenn das Publikum etwas lustig findet und lacht, lässt man zum Beispiel eine größere Pause. Bei der bereits erwähnten 
Lesung „Pussy Riots“ habe ich zum Beispiel die Passage von Lady Bitch Ray mit dem Tennisstöhnkonzert gemacht. Dabei habe ich es geschafft, dass der ganze Saal mit mir gestöhnt hat. Hätten die aber nicht mitgemacht und ich hätte 30 Sekunden probiert, sie zu animieren, dann hätte ich das irgendwann fallen lassen. Sie haben aber mitgemacht und wir haben dann eineinhalb Minuten zusammen gestöhnt und das Publikum hat sich kaputtgelacht. Wenn man hingegen vor einem Mikrofon im Studio sitzt und eine Aufnahme macht, ist es eine ganz andere Situation, der Rhythmus beispielsweise ändert sich einfach.

JS: Was ist Deines Erachtens das Wichtigste für eine gelungene Lesung? - Es können auch mehrere Attribute sein. Ist es beispielsweise die Stimme oder geht es mehr um Attribute des Sprechens, wie Betonung, Lautstärke, Geschwindigkeit o.ä., falls man das überhaupt trennen kann? Geht es vielleicht sogar um das „Korn der Stimme“ (Roland Barthes), um den performativen Überschuss im Sprechen? Oder eher um eine Etablierung einer bestimmten Rolle im Sprechen? Ist die Identifikation mit der gesprochenen Figur oder eher die Distanz zu ihr entscheidend? Oft wird ja fast automatisch die Stimme der Sprecherin/des Sprechers mit der gesprochenen Figur gleichgesetzt, was zum Beispiel bei der Oral Poetry bzw. Hörlyrik, wie sie in diesem Sammelband bei Claudia Benthien und Wiebke Vorrath verhandelt wird, eine zentrale Rolle spielt. Was würdest Du sagen, was ist für Dich der springende Punkt an einer gelungenen Lesung? Was macht das Faszinosum Hörbuch aus?

LL: Abhängig vom Text und der Inszenierung, vor allem die richtige Dosis. Was davon abhängt, was gemacht wird und wie es gemacht wird. Und es ist ab einem gewissen Punkt dann immer auch Geschmackssache. Es hängt dann immer davon ab: Für wen macht man das und wie will man es machen? Man kann natürlich zum Beispiel den Text von Olympe de Gouges „Erklärung der Rechte der Frau und Bürgerin“" wahnsinnig pathetisch oder polemisch sprechen - oder man kann ihn einfach, quasi neutral, zur Verfügung stellen. Das macht einen ganz großen Unterschied in der Sprechhaltung aus. Ich kann die Frage des Textes so stellen, als würde ich als Sprecherin sie jemanden persönlich stellen, aber ich kann sie auch einfach in den Raum stellen und mich zurücknehmen.

JS: Im Text von Katharina Rost in diesem Sammelband analysiert sie das Hörbuch „Games of Thrones - Das Lied von Eis und Feuer“. Es wird von Reinhard Kuhnert gesprochen und er spricht mit einer großen Variabilität. Jede Figur bekommt eine besondere Stimme, was bei der Vielzahl an Charakteren wirklich eine Herausforderung ist, und sicher wiederholen sich bestimmte Eigenschaften auch; letztlich lässt sich die Sprechweise vielleicht auf bestimmte Sprech- und Hörkli- 
schees reduzieren, die er benutzt. Aber dennoch würden viele Zuhörende diese Hörbuchfassung als eine gelungene Lesung bezeichnen.

LL: Was Kuhnert zumindest sehr besonders macht, ist, dass er eben nicht vollständig in Stereotype eintaucht, sondern dass er diese natürlich auch benutzt oder sich an ihnen anlehnt, aber dass er minimale Verstellungen in der Stimme vornimmt und einem keine brachialen Klischees präsentiert, wie - tiefe, kratzige, leicht gehauchte Stimme -: „Ich bin der Böse.“

JS: Es gibt auch ein linguistisches Projekt von Kati Hannken-Illjes, Barbara Schlücker und Nicole Dehé, das im Rahmen dieses Hörbuch-Projekts durchgeführt wurde mit SchülerInnen der 8. Klasse zu ihrem Lese- und Hörverständnis. Der Aufsatz dazu ist ebenfalls in diesem Sammelband publiziert. Bei diesem Projekt hast Du eine große Rolle gespielt, nicht so sehr für den Text in geschriebener Form, sondern vielmehr in gesprochener Form. Du hast nämlich die Texte von Kleist und Keller eingelesen. Kannst Du kurz sagen, worum es in diesem Projekt ging? Beziehungsweise, was war Deine Aufgabe und was hast Du für Erfahrungen machen können?

LL: Es war ein Projekt zur Frage, was SchülerInnen verstehen, wenn sie einen literarischen Text eigenständig still lesen und was sie verstehen, wenn sie diesen hören. Und was ihnen mehr Spaß macht und sie letztlich stärker motiviert, weiter mit den Texten umzugehen, das Lesen oder Hören. Meine Aufgabe war einfach, von beiden Texten einen zehnminütigen Ausschnitt einzulesen. Weiter war das nicht definiert. Der Rest war mir total frei überlassen. Der Unterschied bei diesen beiden Texten ist zum Beispiel, dass Kleist viel längere, komplexere und verschachteltere Sätze schreibt als Keller. Und als Sprecherin ist dann die Herausforderung, die Sprechspannung so weit zu halten, den gedanklichen Bogen so weit zu spannen und am Anfang des Satzes schon zu wissen, worauf es am Ende hinausläuft und nicht zwischendurch in der Sprechspannung herunter zu fallen, weil es dann unverständlich wird. Das ist ganz essentiell bei solchen Texten, wenn man sie einliest, weil sonst der Hörer beim einmaligen Hören gar nicht begreifen kann, worum es geht. Vor allem auch, weil wir es nicht mehr gewohnt sind, so komplexe Sätze zu hören. Und bei Keller sind die Sätze wesentlich leichter, dafür gab es da viele Figuren. Da konnte ich mich mal ein bisschen ausprobieren im Figuren-Sprechen und stimmliche Variationen zur Charakterisierungen einsetzen, also im Sprechtempo, in der Lautstärke oder Tonhöhe variieren. 
JS: Wie geht es dir denn selbst, findest du eher einen Zugang zu Texten über das Lesen oder über das Hören?

LL: Also für die Arbeit, für das Theater oder auch für Drehs oder Ähnliches lese ich. Ganz eindeutig, ich lese und ich lese still. Der erste Arbeitsschritt ist meistens einfach ein stilles Lesen und dann folgt irgendwann etwas laut Gelesenes und dann eine Leseprobe mit den Kollegen, bei der man dann miteinander hört: Wie klingt das? Und auch nochmal neue Eindrücke vom Text bekommt. Im Verlauf einer Arbeit geht die Auseinandersetzung mit dem Text natürlich immer weiter. Aber immer wieder Lesen. Lesen oder selbst hören, was man selbst produziert, so zum Ausprobieren. Für die Arbeit orientiere ich mich gar nicht an eingelesenen Texten. Obwohl es natürlich wahnsinnig tolle Kollegen gibt, die wahnsinnig tolle Sachen gemacht haben: Fritz Kortner, wie er den Shylock spricht - da gibt es auf YouTube ein Video, das ist schon sehr beeindruckend. Aber das sind oft eher Inspirationsquellen. Ich versuche, immer wenn ich selbst an etwas arbeite, mich nicht von anderen Interpretationen leiten $\mathrm{zu}$ lassen, sondern meine eigene, meine einzigartige Umsetzung von einem Text zu finden. Privat höre ich aber gerne Hörbücher.

JS: Nach deiner Erfahrung am Schauspiel Essen, wo Du derzeit arbeitest, und im Theater Erlangen: welchen Status hat das gelesene Wort oder eben die Lesung im Kontext eines Repertoires von Schauspielhäusern? Ist sie häufiger vertreten, ein populäres Genre, oder spielt sie eher eine untergeordnete Rolle?

LL: Tatsächlich gibt es viele Lesungen. Allein die Matineen sind oft Lesungen mit den Texten, die dann zur Premiere gebracht werden. Aber auch zusätzlich werden viele Lesungen produziert. In Erlangen gab es eine ganze Reihe. Das stand damals unter dem Thema „Mensch - Maschine“, da habe ich auch zwei gemacht, „Der Sandmann“ von E.T.A. Hoffmann und „Dr. Jekyll und Mr. Hyde“. Und in Essen gibt es ein Format, das nennt sich „Freischuss“, da können Assistenten jedweder Richtung, also Ausstattungsassistenten, Regieassistenten, Dramaturgieassistenten, wer immer möchte, einen kleinen Abend gestalten, der dann zunächst nur einmal gezeigt wird und wenn er besonders gut läuft, dann wird er auch nochmal gezeigt. Da gibt es auch viele Lesungen, ich habe zum Beispiel drei gemacht: „Die wartende Prinzessin“, „Back to no Future“ und neulich „Pussy Riots“. Die Lesungen sind wahnsinnig gut besucht.

JS: Wie erklärst Du Dir das Interesse an den Lesungen? Weil sie stärker aktuelle Texte und damit Zeitgeistthemen aufgreifen als das klassische Repertoire im Schauspiel? 
LL: Erst einmal finden Lesungen oft in kleineren Räumen statt. Das heißt, ,wahnsinnig gut besucht' ist dann im Vergleich zum großen Haus eher relativ. Oft braucht eine Lesung aber auch einen intimeren Rahmen, ebenso ist der Raum entscheidend: Lesungen auf einer großen Bühne sind schon wieder eine ganz andere Inszenierung als in der Heldenbar oder in der Box usw. Auf einer Kammerbühne kommuniziert man viel direkter mit dem Publikum. Und bei dramatischen Inszenierungen auf der großen Bühne gibt es viel häufiger die Situation, dass man noch mit vierter Wand spielt. Das ist bei Lesungen eher selten.

JS: Man könnte das Ganze natürlich auch in einen größeren Kontext einbetten, es gibt in unserer Kultur in den letzten Jahren ja durchaus eine Hinwendung zum Akustischen, im wissenschaftlichen Kontext wird vom ,,acoustic turn“(Petra Maria Meyer) gesprochen. Zum Beispiel etablieren sich Hörcafés oder Dunkelrestaurants, Livehörspiele ziehen ein vermehrtes Interesse auf sich usw. Erlebt das gemeinsam geteilte, das öffentliche Hören von Geschichten - statt das einsame, private Hören - eine Art Renaissance?

LL: Ja, das kann gut sein, dass das in so eine Richtung geht. Ich glaube aber, dass das, was die Leute zu solchen Veranstaltungen zieht, weniger die Lesung ist, sondern vielmehr das Thema, das angesprochen wird. Also, wenn man Dr. Jekyll und Mr. Hyde macht, dann interessiert sie das. Und genauso ist das aber auch bei Inszenierungen. Das sind andere Sachen, die ziehen, weniger das Format. Das ist ein Thema oder Stück, das zieht, das ist ein Regisseur oder ein Schauspieler, der zieht.

JS: Kommen wir zu einem ganz anderen Punkt: Wie arbeitest Du an einem zu lesenden Text?

LL: Also, wenn man an einer Lesung arbeitet, ist das wahrscheinlich mehr Arbeit als die meisten Leute sich vorstellen. Auch wenn man das Papier in der Hand hat und der Text da gedruckt steht, geht da schon ordentlich Zeit rein, weil man versucht, einen Text zu strukturieren und eine bestimmte Haltung zu ihm zu finden. Ihn auf eine ganz persönliche Art auch irgendwie zu transportieren. Wie auch immer die sein mag. Und das ist vielleicht das, was oft unterschätzt wird; und deshalb aber vielleicht auch der Grund, warum ich den Unterschied so schwer fassen kann zwischen Lesung und Spielen auf der Bühne, weil die Auseinandersetzung bei beidem eine ganz intensive ist.

JS: Und wie gestaltest Du Text, den Du vorliest? Also, markierst Du Dir den Text irgendwie graphisch? Oder merkst Du Dir alles? 
LL: Viele Sachen merkt man sich tatsächlich einfach beim Erarbeiten. Und dann gibt's manche Stellen, an denen stolpert man immer wieder. An denen ist es super wichtig, dass man da dann den Gedankenbogen weiterspannt. Und ich persönlich habe mir ein ganz geheimes Zeichensystem erarbeitet, das nur ich lesen kann: irgendwelche Punkte, Striche, Ausrufezeichen, Pausenzeichen oder kleine Wölkchen und ich weiß dann, was das bedeutet. Ich benutze tatsächlich viele Zeichen aus der Musik. Und das schreibe ich mit Bleistift rein, weil sich so etwas auch immer mal ändert und dann kann man das wegradieren. An der Stelle, wo ich tatsächlich mit Farben arbeite, das sind Momente an denen ich weiß: An dieser Stelle, den Satz weiß ich auswendig und den möchte ich tatsächlich ins Publikum sprechen. Die Stelle danach, die markiere ich mir oft mit Farbe, um sie im Text wieder zu finden.

JS: Eine abschließende Frage: gibt es denn einen sehr bekannten Sprecher oder sehr bekannte Sprecherin, die Du gerne hörst?

LL: Ich höre gerne Katharina Thalbach. Ich kenne auch viele Leute, die sie ganz fürchterlich finden. Ich mag die Körnigkeit ihrer Stimme, ich mag dieses Brüchige, was da drin ist und ich finde, dass sie wahnsinnig gut erzählt.

JS: Ja, das fällt mir ohnehin auf, dass beliebte Stimmen auch immer ganz radikal unbeliebte Stimmen sind. Also die ,großen 'Stimmen scheinen mir immer welche zu sein, die zu großen Kontroversen aufrufen, die aber eben diese besondere Auffälligkeit erlangen. Sophie Rois ist dafür auch ein gutes Beispiel. In diesem Sinne, Lisan, ich danke Dir ganz herzlich für das Gespräch. 
